\title{
The Equivalence of Operator Norm between the Hardy-Littlewood Maximal Function and Truncated Maximal Function on the Heisenberg Group
}

\author{
Xiang Li $\mathbb{D}^{1}$ and Xingsong Zhang $\mathbb{D}^{2}$ \\ ${ }^{1}$ School of Mathematics and Finance, Chuzhou University, Chuzhou, Anhui 239012, China \\ ${ }^{2}$ RDFZ Chaoyang School, Beijing 100028, China
}

Correspondence should be addressed to Xingsong Zhang; zhangxingsong17@mails.ucas.ac.cn

Received 5 June 2021; Revised 13 July 2021; Accepted 6 August 2021; Published 24 August 2021

Academic Editor: Andrea Scapellato

Copyright (c) 2021 Xiang Li and Xingsong Zhang. This is an open access article distributed under the Creative Commons Attribution License, which permits unrestricted use, distribution, and reproduction in any medium, provided the original work is properly cited.

In this article, we define a kind of truncated maximal function on the Heisenberg space by $\mathbb{M}_{\gamma}^{c} f(x)=\sup _{0<r<\gamma}(1 / m(B(x, r))) \int_{B(x, r)}|f(y)| d y$. The equivalence of operator norm between the Hardy-Littlewood maximal function and the truncated maximal function on the Heisenberg group is obtained. More specifically, when $1<p<\infty$, the $L^{p}$ norm and central Morrey norm of truncated maximal function are equal to those of the Hardy-Littlewood maximal function. When $p=1$, we get the equivalence of weak norm $L^{1} \longrightarrow$ $L^{1, \infty}$ and $\dot{M}^{1, \lambda} \longrightarrow \dot{W} M^{1, \lambda}$. Those results are generalization of previous work on Euclid spaces.

\section{Introduction}

Let $f$ be a locally integrable function on $\mathbb{R}^{n}$. We define the centered Hardy-Littlewood maximal function as

$$
M^{c} f(x)=\sup _{r>0} \frac{1}{m(B(x, r))} \int_{B(x, r)}|f(y)| d m(y),
$$

and define the uncentered Hardy-Littlewood maximal function as

$$
M f(x)=\sup _{B \ni x} \frac{1}{m(B)} \int_{B}|f(y)| d m(y) .
$$

The Hardy-Littlewood maximal functions play an important role in harmonic analysis. Their boundness and sharp bounds are important since a variety of operators are controlled by maximal functions. The $L^{1} \longrightarrow$ $L^{1, \infty}$ and $L^{p} \longrightarrow L^{p}$ boundness of Hardy-Littlewood maximal functions are well-known [1-5]. However, sharp bounds are very hard to obtain. For a long time, we only know how the sharp bounds of Hardy-Littlewood maximal functions behave when the dimension $n$ changes. In 2003, Melas [6] obtained the sharp bound of the one-dimensional centered Hardy-Littlewood maximal function of weak type $(1,1)$. But it is hard to apply his method to higher dimensional cases. No result has been stated for general cases.

Now, we introduce another point of view. In [7], Wei et al. defined a truncated maximal function:

Definition 1. Let $f \in L_{l o c}\left(\mathbb{R}^{n}\right)$ be a locally integrable function on $\mathbb{R}^{n}$. We define truncated centered maximal function:

$$
M_{\alpha, \beta}^{c} f(x)=\sup _{\alpha<r<\beta} \frac{1}{m(B(x, r))} \int_{B(x, r)}|f(y)| d y,
$$

and truncated uncentered maximal function:

$$
M_{\alpha, \beta} f(x)=\sup _{B \ni x, \alpha<\operatorname{radi}(B)<\beta} \frac{1}{m(B)} \int_{B}|f(y)| d y,
$$


where $\operatorname{radi}(B)$ is the radium of ball $B .0 \leq \alpha<\beta \leq \infty . \beta=\infty$ means that there is no limit of upper bound of $r$ or $\operatorname{radi}(B)$.

When $\alpha=0$, we write truncated centered maximal function briefly as

$$
M_{\gamma}^{c} f(x)=\sup _{0<r<\gamma} \frac{1}{m(B(x, r))} \int_{B(x, r)}|f(y)| d y .
$$

Then, we have the following theorem [7]:

Theorem 2. For $\gamma>0$, there is

$$
\left\|M^{c}\right\|_{L^{1}\left(\mathbb{R}^{n}\right) \longrightarrow L^{1, \infty}\left(\mathbb{R}^{n}\right)}=\left\|M_{\gamma}^{c}\right\|_{L^{1}\left(\mathbb{R}^{n}\right) \longrightarrow L^{1, \infty}\left(\mathbb{R}^{n}\right)} .
$$

For $1<p \leq \infty, \gamma>0$, there is

$$
\left\|M^{c}\right\|_{L^{p}\left(\mathbb{R}^{n}\right) \longrightarrow L^{p}\left(\mathbb{R}^{n}\right)}=\left\|M_{\gamma}^{c}\right\|_{L^{p}\left(\mathbb{R}^{n}\right) \longrightarrow L^{p}\left(\mathbb{R}^{n}\right)}
$$

There holds similar results of truncated uncentered maximal function. We also can prove that the operator norm of the truncated maximal function with $(\alpha, \beta)$ is only related to $C$ $=\alpha / \beta$. When $C$ tends to zero, the norm tends to that of the Hardy-Littlewood maximal function.

We choose to study the truncated maximal operator and maximal operator because there are some differences among them while their operator norms are equivalent. For example, for locally integrable functions with compact support, their truncated maximal functions have compact support while their Hardy-Littlewood maximal functions may not have. Based on the above reasons, it will be easier to study the truncated maximal functions sometimes. We hope that equivalence and difference among truncated maximal operator and maximal operator may bring new thoughts and methods.

In addition, Zhang et al. obtained the equivalence of operator norm between the truncated maximal function and the Hardy-Littlewood function on Morrey spaces in [8]:

Definition 3. Set $1 \leq q<\infty$ and $0 \leq \lambda \leq n$. For $f \in L_{l o c}^{q}\left(\mathbb{R}^{n}\right)$, define the central Morrey norm $\|f\|_{\dot{M}^{q, \lambda}\left(\mathbb{R}^{n}\right)}$ :

$$
\|f\|_{\dot{M}^{q, \lambda}\left(R^{n}\right)}=\sup _{R>0}\left(\frac{1}{R^{\lambda}} \int_{B(0, R)}|f(x)|^{q} d x\right)^{1 / q}
$$

where $B(0, R)$ is a ball centered in origin with radium $R$.

The central Morrey space $\dot{M}^{q, \lambda}\left(\mathbb{R}^{n}\right)$ is defined as

$$
\dot{M}^{q, \lambda}\left(\mathbb{R}^{n}\right)=\left\{f \in L_{l o c}^{q}\left(\mathbb{R}^{n}\right):\|f\|_{\dot{M}^{q, \lambda}\left(\mathbb{R}^{n}\right)}<\infty\right\} .
$$

Set $1 \leq q<\infty$ and $0 \leq \lambda \leq n$. For any measurable function $f$, define weak the central Morrey norm $\|f\|_{\dot{W} M^{q, \lambda}\left(\mathbb{R}^{n}\right)}$ :

$$
\|f\|_{\dot{W} M^{q, \lambda}\left(\mathbb{R}^{n}\right)}=\sup _{R>0} \frac{1}{R^{\lambda / q}} \sup _{s>0} s|\{y \in B(0, R):|f(y)|>s\}|^{1 / q} .
$$

The weak centered Morrey space $\dot{W} M^{q, \lambda}\left(\mathbb{R}^{n}\right)$ is defined as

$\dot{W} M^{q, \lambda}\left(\mathbb{R}^{n}\right)=\left\{\right.$ measurable function $\left.f:\|f\|_{\dot{W} M^{q, \lambda}\left(\mathbb{R}^{n}\right)}<\infty\right\}$.

Zhang et al. proved the following theorem [8]:

Theorem 4. For $0<\lambda<n, \gamma>0$, there is

$$
\left\|M^{c}\right\|_{\dot{M}^{1, \lambda}\left(\mathbb{R}^{n}\right) \longrightarrow \dot{W} M^{1, \lambda}\left(\mathbb{R}^{n}\right)}=\left\|M_{\gamma}^{c}\right\|_{\dot{M}^{1, \lambda}\left(\mathbb{R}^{n}\right) \longrightarrow \dot{W} M^{1, \lambda}\left(\mathbb{R}^{n}\right)} .
$$

For $1<p<\infty, 0<\lambda<n, \gamma>0$, there is

$$
\left\|M^{c}\right\|_{\dot{M}^{p, \lambda}\left(\mathbb{R}^{n}\right) \longrightarrow \dot{M}^{p, \lambda}\left(\mathbb{R}^{n}\right)}=\left\|M_{\gamma}^{c}\right\|_{\dot{M}^{p, \lambda}\left(\mathbb{R}^{n}\right) \longrightarrow \dot{M}^{p, \lambda}\left(\mathbb{R}^{n}\right)} .
$$

Those are the results on $\mathbb{R}^{n}$. Nowdays, the researchers concern about the classic operators on more abstract background such as p-adic fields and the Heisenberg group. Some researchers have already obtained the boundness of the Hardy-Littlewood maximal function on the Heisenberg group, one example is the following theorem in [9]:

Theorem 5. There exists constant $A$ such that for $\ln n \gg 1$, we have

$$
\left\|\mathbb{M}^{c} f\right\|_{L^{1, \infty}\left(\mathbb{H}^{n}\right)} \leq A n\|f\|_{L^{1}\left(\mathbb{H}^{n}\right)} .
$$

We can see that there is still no specific sharp bound. In order to apply the methods of truncated maximal functions, we are going to establish the equivalence of operator norm between the Hardy-Littlewood maximal functions and truncated maximal functions on the Heisenberg group.

We outline some basic information of the Heisenberg group. The Heisenberg group $\mathbb{H}^{n}$ is underlying manifold $\mathbb{R}^{2 n} \times \mathbb{R}^{1}$ with group law

$$
\begin{aligned}
& \left(x_{1}, x_{2}, \cdots, x_{2 n}, x_{2 n+1}\right)\left(x_{1}^{\prime}, x_{2}^{\prime}, \cdots, x_{2 n}^{\prime}, x_{2 n+1}^{\prime}\right) \\
& \quad=\left(x_{1}+x_{1}^{\prime}, x_{2}+x_{2}^{\prime}, \cdots, x_{2 n}+x_{2 n}^{\prime}, x_{2 n+1}+x_{2 n+1}^{\prime}+2 \sum_{j=1}^{n} x_{j}^{\prime} x_{n+j}-x_{j} x_{n+j}^{\prime}\right) .
\end{aligned}
$$

The identity element on $\mathbb{H}^{n}$ is $0 \in \mathbb{R}^{2 n+1}$, and the inverse element $x^{-1}$ is $-x$. The Haar measure on $\mathbb{H}^{n}$ coincides with the Lebesgue measure on $\mathbb{R}^{2 n} \times \mathbb{R}^{1}$. 
$\mathbb{H}^{n}$ is a homogeneous group with dilations

$$
\delta_{r}\left(x_{1}, x_{2}, \cdots, x_{2 n}, x_{2 n+1}\right)=\left(r x_{1}, r x_{2}, \cdots, r x_{2 n}, r^{2} x_{2 n+1}\right), r>0 \text {. }
$$
Then

Set $|E|$ as the measure of any measurable set $E$ in $\mathbb{H}^{n}$.

$$
\begin{aligned}
& \left|\delta_{r}(E)\right|=r^{Q}|E|, \\
& d\left(\delta_{r} x\right)=r^{Q} d x,
\end{aligned}
$$

where $Q=2 n+2$ is called the homogeneous dimension of $\mathbb{H}^{n}$.

The metric on $\mathbb{H}^{n}$ derived from the norm

$$
|x|_{h}=\left[\left(\sum_{i=1}^{2 n} x_{i}^{2}\right)+x_{2 n+1}^{2}\right]^{1 / 4},
$$

where $x=\left(x_{1}, x_{2}, \cdots, x_{2 n}, x_{2 n+1}\right)$, is given by

$$
d(p, q)=d\left(q^{-1} p, 0\right)=\left|q^{-1} p\right|_{h}
$$

For $r>0$ and $x \in \mathbb{H}^{n}$, define the ball and sphere with center $x$ and radius $r$ on $\mathbb{H}^{n}$ as

$$
\begin{aligned}
& B(x, r)=\left\{y \in \mathbb{H}^{n}: d(x, y)<r\right\}, \\
& S(x, r)=\left\{y \in \mathbb{H}^{n}: d(x, y)=r\right\} .
\end{aligned}
$$

We also have

$$
|B(x, r)|=|B(0, r)|=\Omega_{Q} r^{Q}
$$

where

$$
\Omega_{Q}=\frac{2 \pi^{n+1 / 2} \Gamma(n / 2)}{(n+1) \Gamma(n) \Gamma((n+1) / 2)} .
$$

$\Omega_{Q}$ is the volume of the unit ball $B(0,1)$ on $\mathbb{H}^{n}$, and the area of the sphere $S(0,1)$ is $\omega_{Q}=Q \Omega_{Q}$.

For further information, readers could refer to [9].

Now, we can present the operators on the Heisenberg group.

For $f \in L_{l o c}\left(\mathbb{H}^{n}\right)$, we define the centered HardyLittlewood maximal function on the Heisenberg group:

$$
\mathbb{M}^{c} f(x)=\sup _{r>0} \frac{1}{|B(x, r)|} \int_{B(x, r)}|f(y)| d y,
$$

and a kind of truncated maximal function on the Heisenberg group:

$$
\mathbb{M}_{\gamma}^{c} f(x)=\sup _{0<r<\gamma} \frac{1}{m(B(x, r))} \int_{B(x, r)}|f(y)| d y .
$$

We will establish equivalence of their operator norms on the Heisenberg group. In the next section, we introduce some preliminaries and basic lemmas. In the third section, we present the details of our main theorems and the proofs.

\section{Preliminaries}

In this section, we present some preliminaries and basic lemmas.

First, we give the definition of distribution function on the Heisenberg group (similar to that on $\mathbb{R}^{n}$ ):

Definition 6. Let $f$ be a measurable function on $\mathbb{H}^{n}$. Define distribution function $d_{f}: \mathbb{H}^{n} \longrightarrow[0,+\infty)$ as

$$
d_{f}(\alpha)=\left|\left\{x \in \mathbb{H}^{n}:|f(x)|>\alpha\right\}\right|,
$$

where $|*|$ is the Lebesgue measure on $\mathbb{R}^{2 n+1}$.

We have such a relation between distribution function and $L^{p}$ norms:

Lemma 7. Set $0<p<\infty$ and $f \in L^{p}\left(\mathbb{H}^{n}\right)$. Then, we have

$$
\|f\|_{L^{p}\left(\mathbb{H}^{n}\right)}^{p}=p \int_{0}^{\infty} \alpha^{p-1} d_{f}(\alpha) d \alpha
$$

This lemma is a basic equivalent expression of $L^{p}$ norm, and readers can find the proof in Proposition 1.1.4 of [3].

Lemma 8. Let $\mu$ be a positive measure on $\sigma$-algebra $\mathbb{M}$. If sets $\left\{A_{n}\right\}$ satisfy that $A_{1} \subset A_{2} \subset A_{3} \cdots \subset A_{n} \cdots$, and $A=\cup_{n=1}^{\infty} A_{n}$, then we have

$$
\lim _{n \rightarrow \infty} \mu\left(A_{n}\right)=\mu(A)
$$

Readers can find proofs of Lemma 8 in Theorem 1.19 (d) of [10].

Using Lemma 8, we can obtain the following lemma.

Lemma 9. Let $\mathbb{M}^{c}$ and $\mathbb{M}_{\gamma}^{c}$ be the operators defined in (23) and (24). Then, for any $f \in L^{p}\left(\mathbb{H}^{n}\right)$ and $\lambda>0$, we have

$$
d_{\mathbb{M}^{c} f}(\lambda)=\lim _{\gamma \longrightarrow \infty} d_{\mathbb{M}_{\gamma}^{c} f}(\lambda)
$$

Proof. Fix $x \in \mathbb{H}^{n}$. For any $\varepsilon$, there exists $r_{\varepsilon}>0$ such that

$$
\frac{1}{m\left(B\left(x, r_{\varepsilon}\right)\right)} \int_{B\left(x, r_{\varepsilon}\right)}|f(y)| d y>\mathbb{M}^{c} f(x)-\varepsilon
$$

For some large $\gamma$, we have

$$
\mathbb{M}_{\gamma}^{c} f(x) \geq \frac{1}{m\left(B\left(x, r_{\varepsilon}\right)\right)} \int_{B\left(x, r_{\varepsilon}\right)}|f(y)| d y>\mathbb{M}^{c} f(x)-\varepsilon
$$


Since $\mathbb{M}_{\gamma}^{c} f(x)$ increases as $\gamma$ becomes larger, then

$$
\lim _{\gamma \longrightarrow \infty} \mathbb{M}_{\gamma}^{c} f(x) \geq \mathbb{M}^{c} f(x)
$$

On the other hand, by definition we have

$$
\mathbb{M}_{\gamma}^{c} f(x) \leq \mathbb{M}^{c} f(x)
$$

Then

$$
\lim _{\gamma \longrightarrow \infty} \mathbb{M}_{\gamma}^{c} f(x)=\mathbb{M}^{c} f(x) .
$$

Set $k=1,2,3, \cdots$ :

$$
\begin{gathered}
A_{k}=\left\{x \in: \mathbb{M}_{k}^{c} f(x)>\lambda\right\}, \\
A=\left\{x \in: \mathbb{M}^{c} f(x)>\lambda\right\} .
\end{gathered}
$$

By (33) and Lemma 8, we obtain that

$$
d_{\mathbb{M}^{c} f}(\lambda)=|A|=\lim _{k \longrightarrow \infty}\left|A_{k}\right|=\lim _{k \longrightarrow \infty} d_{M_{k}^{c} f}(\lambda)=\lim _{\gamma \longrightarrow \infty} d_{M_{\gamma}^{c} f}(\lambda) .
$$

Lemma 9 implies that when $\gamma$ tends to infinite, the distribution tends to equal. It will be important in the proof of weak-type operator norms. For strong-type operator norms, we have the following lemma:

Lemma 10. $1<p<\infty$. Fixing $\varepsilon>0$, there is a function $g \in$ $C_{c}^{\infty}\left(\mathbb{H}^{n}\right)$ such that

$$
\frac{\left\|\mathbb{M}^{c} g\right\|_{L^{p}\left(\mathbb{H}^{n}\right)}}{\|g\|_{L^{p}\left(\mathbb{H}^{n}\right)}} \geq\left\|\mathbb{M}^{c}\right\|_{L^{p}\left(\mathbb{H}^{n}\right) \longrightarrow L^{p}\left(\mathbb{H}^{n}\right)}-\varepsilon .
$$

Proof. By the definition of operator norm, there exists a function $f \in L^{p}\left(\mathbb{H}^{n}\right)$ such that

$$
\frac{\left\|\mathbb{M}^{c} f\right\|_{L^{p}\left(\mathbb{H}^{n}\right)}}{\|f\|_{L^{p}\left(\mathbb{H}^{n}\right)}} \geq\left\|\mathbb{M}^{c}\right\|_{L^{p}\left(\mathbb{H}^{n}\right) \longrightarrow L^{p}\left(\mathbb{H}^{n}\right)}-\varepsilon / 2 .
$$

Since $C_{c}^{\infty}\left(\mathbb{H}^{n}\right)$ is dense in $L^{p}\left(\mathbb{H}^{n}\right)$, then we have $g \in C_{c}^{\infty}$ $\left(\mathrm{H}^{n}\right)$ such that

$$
\|f-g\|_{L^{p}\left(\mathbb{H}^{n}\right)}<\delta
$$

Let $A$ be a upper bound of norm of $\mathbb{M}^{c}$. Then

$$
\left\|\mathbb{M}^{c}(f-g)\right\|_{L^{p}\left(\mathbb{H}^{n}\right)}<A\|f-g\|_{L^{p}\left(\mathbb{H}^{n}\right)}<A \delta .
$$

So

$$
\begin{aligned}
\frac{\left\|\mathbb{M}^{c} g\right\|_{L^{p}\left(\mathbb{H}^{n}\right)}}{\|g\|_{L^{p}\left(\mathbb{H}^{n}\right)}} & \geq \frac{\left\|\mathbb{M}^{c} f\right\|_{L^{p}\left(\mathbb{H}^{n}\right)}-\left\|\mathbb{M}^{c}(f-g)\right\|_{L^{p}\left(\mathbb{H}^{n}\right)}}{\|f\|_{L^{p}\left(\mathbb{H}^{n}\right)}+\|f-g\|_{L^{p}\left(\mathbb{H}^{n}\right)}} \\
& \geq \frac{\left\|\mathbb{M}^{c} f\right\|_{L^{p}\left(\mathbb{H}^{n}\right)}-A \delta}{\|f\|_{L^{p}\left(\mathbb{H}^{n}\right)}+\delta} .
\end{aligned}
$$

Pick suitable $\delta$ in order that

$$
\begin{aligned}
\frac{\left\|\mathbb{M}^{c} f\right\|_{L^{p}\left(\mathbb{H}^{n}\right)}-A \delta}{\|f\|_{L^{p}\left(\mathbb{H}^{n}\right)}+\delta} & \geq \frac{\left\|\mathbb{M}^{c} f\right\|_{L^{p}\left(\mathbb{H}^{n}\right)}}{\|f\|_{L^{p}\left(\mathbb{H}^{n}\right)}}-\varepsilon / 2 \\
& \geq\left\|\mathbb{M}^{c}\right\|_{L^{p}\left(\mathbb{H}^{n}\right) \longrightarrow L^{p}\left(\mathbb{H}^{n}\right)}-\varepsilon .
\end{aligned}
$$

Then, we finish the proof.

Notice that $g$ has compact support. This property is important when we establish equivalence of operator norms.

We need the following key lemma:

Lemma 11. Let $\gamma>0$. Then

$$
\mathbb{M}_{\gamma}^{c} f\left(\delta_{\gamma} x\right)=\mathbb{M}_{1}^{c}\left(\tau_{\gamma} f\right)(x)
$$

where $\tau_{\gamma} f(x)=f\left(\delta_{\gamma} x\right)$.

Proof. By direct computation, we arrive at

$$
\begin{aligned}
\mathbb{M}_{\gamma}^{c} f(\gamma x) & =\sup _{0<r<\gamma} \frac{1}{\Omega_{Q} r^{Q}} \int_{|y|<r}\left|f\left(\delta_{\gamma} x-y\right)\right| d y \\
& =\sup _{0<r<\gamma} \frac{\gamma^{Q}}{\Omega_{Q} r^{Q}} \int_{|y|<r / \gamma}\left|f\left(\delta_{\gamma} x-\delta_{\gamma} y\right)\right| d y \\
& =\sup _{0<r / \gamma<1} \frac{1}{\Omega_{Q}(r / \gamma)^{Q}} \int_{|y|<r / \gamma}\left|\left(\tau_{\gamma} f\right)(x-y)\right| d y \\
& =\mathbb{M}_{1}^{c}\left(\tau_{\gamma} f\right)(x) .
\end{aligned}
$$

This lemma is based on dilation. Remind that the dilation on the Heisenberg group is different from that on Euclid spaces.

Notice that Lemma 11 implies the relation between $\mathbb{M}_{\gamma}^{c}$ and $\mathbb{M}_{1}^{c}$. Connecting it with the definition of operator norm, we obtain the following Lemma 12:

\section{Lemma 12.}

(1) For $\gamma>0$, we have

$$
\left\|\mathbb{M}_{1}^{c}\right\|_{L^{1}\left(\mathbb{H}^{n}\right) \longrightarrow L^{1, \infty}\left(\mathbb{H}^{n}\right)}=\left\|\mathbb{M}_{\gamma}^{c}\right\|_{L^{1}\left(\mathbb{H}^{n}\right) \longrightarrow L^{1, \infty}\left(\mathbb{H}^{n}\right)}
$$

(2) For $1<p \leq \infty, \gamma>0$, we have 


$$
\left\|\mathbb{M}_{1}^{c}\right\|_{L^{p}\left(\mathbb{H}^{n}\right) \longrightarrow L^{p}\left(\mathbb{H}^{n}\right)}=\left\|\mathbb{M}_{\gamma}^{c}\right\|_{L^{p}\left(\mathbb{H}^{n}\right) \longrightarrow L^{p}\left(\mathbb{H}^{n}\right)}
$$

(3) For $0<\lambda<n, \gamma>0$, we have

$$
\left\|\mathbb{M}_{1}^{c}\right\|_{\dot{M}^{1, \lambda}\left(\mathbb{H}^{n}\right) \longrightarrow \dot{W} M^{1, \lambda}\left(\mathbb{H}^{n}\right)}=\left\|\mathbb{M}_{\gamma}^{c}\right\|_{\dot{M}^{1, \lambda}\left(\mathbb{H}^{n}\right) \longrightarrow \dot{W} M^{1, \lambda}\left(\mathbb{H}^{n}\right)} .
$$

(4) For $1<p<\infty, 0<\lambda<n, \gamma>0$, we have

$$
\left\|\mathbb{M}_{1}^{c}\right\|_{\dot{M}^{p, \lambda}\left(\mathbb{H}^{n}\right) \longrightarrow \dot{M}^{p, \lambda}\left(\mathbb{H}^{n}\right)}=\left\|\mathbb{M}_{\gamma}^{c}\right\|_{\dot{M}^{p, \lambda}\left(\mathbb{H}^{n}\right) \longrightarrow \dot{M}^{p, \lambda}\left(\mathbb{H}^{n}\right)}
$$

Proof. Based on Lemma 11, we have

(1) For $\lambda>0$

$$
\begin{aligned}
\left|\left\{x: \mathbb{M}_{1}^{c}\left(\tau_{\gamma} f\right)(x)>\lambda\right\}\right| & =\left|\left\{x: \mathbb{M}_{\gamma}^{c} f\left(\delta_{\gamma} x\right)>\lambda\right\}\right| \\
& =\left|\left\{\delta_{\frac{1}{\gamma}} x: \mathbb{M}_{\gamma}^{c} f(x)>\lambda\right\}\right| \\
& =\frac{1}{\gamma^{Q}}\left|\left\{x: \mathbb{M}_{\gamma}^{c} f(x)>\lambda\right\}\right| .
\end{aligned}
$$

Then, there stands

$$
\begin{aligned}
& \frac{1}{\gamma^{\mathrm{Q}}} \frac{\sup _{\lambda>0}\left|\left\{x: \mathbb{M}_{\gamma}^{c} f(x)>\lambda\right\}\right|}{\|f\|_{L^{1}\left(\mathbb{H}^{n}\right)}}=\frac{\sup _{\lambda>0}\left|\left\{x: \mathbb{M}_{1}^{c}\left(\tau_{\gamma} f\right)(x)>\lambda\right\}\right|}{\|f\|_{L^{1}\left(\mathbb{H}^{n}\right)}} \\
& =\frac{1}{\gamma^{Q}} \frac{\sup _{\lambda>0}\left|\left\{x: \mathbb{M}_{1}^{c}\left(\tau_{\gamma} f\right)(x)>\lambda\right\}\right|}{\left\|\tau_{\gamma} f\right\|_{L^{1}\left(\mathbb{H}^{n}\right)}} .
\end{aligned}
$$

Taking the supremum over all $f$ with $\|f\|_{L^{1}\left(\mathbb{H}^{n}\right)}>0$, we have

$$
\left\|\mathbb{M}_{1}^{c}\right\|_{L^{1}\left(\mathbb{H}^{n}\right) \longrightarrow L^{1, \infty}\left(\mathbb{H}^{n}\right)}=\left\|\mathbb{M}_{\gamma}^{c}\right\|_{L^{1}\left(\mathbb{H}^{n}\right) \longrightarrow L^{1, \infty}\left(\mathbb{H}^{n}\right)} .
$$

(2) Using the same method of dilation, it is obvious that

$$
\frac{\left\|\mathbb{M}_{\gamma}^{c} f\right\|_{L^{p}\left(\mathbb{H}^{n}\right)}}{\|f\|_{L^{p}\left(\mathbb{H}^{n}\right)}}=\frac{\left\|\mathbb{M}_{\gamma}^{c} f\left(\delta_{\gamma} \cdot\right)\right\|_{L^{p}\left(\mathbb{H}^{n}\right)}}{\left\|f\left(\delta_{\gamma} \cdot\right)\right\|_{L^{p}\left(\mathbb{H}^{n}\right)}}=\frac{\left\|\mathbb{M}_{1}^{c}\left(\tau_{\gamma} f\right)\right\|_{L^{p}\left(\mathbb{H}^{n}\right)}}{\left\|\tau_{\gamma} f\right\|_{L^{p}\left(\mathbb{H}^{n}\right)}} .
$$

Taking the supremum over all $f$ with $\|f\|_{L^{p}\left(\mathbb{H}^{n}\right)}>0$, we have

$$
\left\|\mathbb{M}_{1}^{c}\right\|_{L^{p}\left(\mathbb{H}^{n}\right) \longrightarrow L^{p}\left(\mathbb{H}^{n}\right)}=\left\|\mathbb{M}_{\gamma}^{c}\right\|_{L^{p}\left(\mathbb{H}^{n}\right) \longrightarrow L^{p}\left(\mathbb{H}^{n}\right)} .
$$

(3) By homogeneous dimension of $\mathbb{H}^{n}$, we obtain that

$$
\|f\|_{\dot{W} M^{1, \lambda}\left(\mathbb{H}^{n}\right)}=\gamma^{Q-\lambda}\left\|\tau_{\gamma} f\right\|_{\dot{W} M^{1, \lambda}\left(\mathbb{H}^{n}\right)} .
$$

There holds that

$$
\begin{aligned}
\left\|\mathbb{M}_{\gamma}^{c} f\right\|_{\dot{W} M^{1, \lambda}\left(\mathbb{H}^{n}\right)} & =\sup _{R>0} \frac{1}{R^{\lambda}} \sup _{s>0} s\left|\left\{y \in B(0, R): \mathbb{M}_{\gamma}^{c} f(y)>s\right\}\right| \\
& =\sup _{R>0} \frac{1}{R^{\lambda}} \sup _{s>0} s \gamma^{Q}\left|\left\{\delta_{\frac{1}{\gamma}} y: y \in B(0, R), \mathbb{M}_{\gamma}^{c} f(y)>s\right\}\right| \\
& =\sup _{R>0} \frac{1}{R^{\lambda}} \sup _{s>0} s \gamma^{Q}\left|\left\{|z|_{h}<\frac{R}{\gamma}: \mathbb{M}_{\gamma}^{c} f\left(\delta_{\gamma} z\right)>s\right\}\right| \\
& =\sup _{R>0} \frac{1}{R^{\lambda}} \sup _{s>0} s \gamma^{Q}\left|\left\{|z|_{h}<\frac{R}{\gamma}: \mathbb{M}_{1}^{c}\left(\tau_{\gamma} f\right)(z)>s\right\}\right| \\
& =\gamma^{Q-\lambda} \sup _{R>0} \frac{1}{(R / \gamma)^{\lambda}} \sup _{s>0} s \gamma^{Q}\left|\left\{|z|_{h}<\frac{R}{\gamma}: \mathbb{M}_{1}^{c}\left(\tau_{\gamma} f\right)(z)>s\right\}\right| \\
& =\gamma^{Q-\lambda} \sup _{R>0} \frac{1}{R^{\lambda}} \sup _{s>0} s \gamma^{Q}\left|\left\{|z|_{h}<R: \mathbb{M}_{1}^{c}\left(\tau_{\gamma} f\right)(z)>s\right\}\right| \\
& =\gamma^{Q-\lambda}\left\|\mathbb{M}_{1}^{c}\left(\tau_{\gamma} f\right)\right\|_{\dot{W} M^{1, \lambda}\left(\mathbb{H}^{n}\right)} .
\end{aligned}
$$

Then

$$
\frac{\left\|\mathbb{M}_{\gamma}^{c} f\right\|_{\dot{W} M^{1, \lambda}\left(\mathbb{H}^{n}\right)}}{\|f\|_{\dot{M}^{1, \lambda}\left(\mathbb{H}^{n}\right)}}=\frac{\left\|\mathbb{M}_{1}^{c}\left(\tau_{\gamma} f\right)\right\|_{\dot{W} M^{1, \lambda}\left(\mathbb{H}^{n}\right)}}{\left\|\tau_{\gamma} f\right\|_{\dot{M}^{1, \lambda}\left(\mathbb{H}^{n}\right)}} .
$$

Taking the spermum over all $f$ with $\|f\|_{\dot{M}^{1, \lambda}\left(\mathbb{H}^{n}\right)}>0$, we have

$$
\left\|\mathbb{M}_{1}^{c}\right\|_{\dot{M}^{1, \lambda}\left(\mathbb{H}^{n}\right) \longrightarrow \dot{W} M^{1, \lambda}\left(\mathbb{H}^{n}\right)}=\left\|\mathbb{\mathbb { M } _ { \gamma } ^ { c }}\right\|_{\dot{M}^{1, \lambda}\left(\mathbb{H}^{n}\right) \longrightarrow \dot{W} M^{1, \lambda}\left(\mathbb{H}^{n}\right)}
$$

(4) By homogeneous dimension of $\mathbb{H}^{n}$, we obtain that

$$
\|f\|_{\dot{M}^{p, \lambda}\left(\mathbb{H}^{n}\right)}=\gamma^{(Q-\lambda) / p}\left\|\tau_{\gamma} f\right\|_{\dot{M}^{p, \lambda}\left(\mathbb{H}^{n}\right)} .
$$


So we get that

$$
\begin{aligned}
\left\|\mathbb{M}_{\gamma}^{c} f\right\|_{\dot{M}^{p, \lambda}\left(\mathbb{H}^{n}\right)}^{p} & =\sup _{R>0}\left(\frac{1}{R^{\lambda}} \int_{B(0, R)}\left|\mathbb{M}_{\gamma}^{c} f(x)\right|^{p} d x\right) \\
& =\sup _{R>0}\left(\frac{1}{R^{\lambda}} \int_{B(0, R / \gamma)}\left|\mathbb{M}_{\gamma}^{c} f\left(\delta_{\gamma} y\right)\right|^{p} \gamma^{Q} d y\right) \\
& =\sup _{R>0}\left(\frac{1}{R^{\lambda}} \int_{B(0, R / \gamma)}\left|\mathbb{M}_{1}^{c}\left(\tau_{\gamma} f\right)(y)\right|^{p} \gamma^{Q} d y\right) \\
& =\gamma^{Q-\lambda} \sup _{R>0}\left(\frac{1}{(R / \lambda)^{\lambda}} \int_{B(0, R / \gamma)}\left|\mathbb{M}_{1}^{c}\left(\tau_{\gamma} f\right)(y)\right|^{p} d y\right) \\
& =\gamma^{Q-\lambda}\left\|\mathbb{M}_{1}^{c}\left(\tau_{\gamma} f\right)\right\|_{\dot{M}^{p, \lambda}\left(\mathbb{H}^{n}\right)}^{p} .
\end{aligned}
$$

Then

$$
\frac{\left\|\mathbb{M}_{\gamma}^{c} f\right\|_{\dot{M}^{p, \lambda}\left(\mathbb{H}^{n}\right)}}{\|f\|_{\dot{M}^{p, \lambda}\left(\mathbb{H}^{n}\right)}}=\frac{\left\|\mathbb{M}_{1}^{c}\left(\tau_{\gamma} f\right)\right\|_{\dot{M}^{p, \lambda}\left(\mathbb{H}^{n}\right)}}{\left\|\tau_{\gamma} f\right\|_{\dot{M}^{p, \lambda}\left(\mathbb{H}^{n}\right)}} .
$$

Taking the spermum over all $f$ with $\|f\|_{\dot{M}^{p, \lambda}\left(\mathbb{H}^{n}\right)}>0$, we have

$$
\left\|\mathbb{M}_{1}^{c}\right\|_{\dot{M}^{p, \lambda}\left(\mathbb{H}^{n}\right) \longrightarrow \dot{M}^{p, \lambda}\left(\mathbb{H}^{n}\right)}=\left\|\mathbb{M}_{\gamma}^{c}\right\|_{\dot{M}^{p, \lambda}\left(\mathbb{H}^{n}\right) \longrightarrow \dot{M}^{p, \lambda}\left(\mathbb{H}^{n}\right)} .
$$

Now, we finish the proof.

Lemma 12 suggests that the operator norm is unrelated to the limit of radius $\gamma$.

\section{Main Theorem}

In this section, we present our main results. We divide them into four theorems:

Theorem 13. For $\gamma>0$, we have

$$
\left\|\mathbb{M}^{c}\right\|_{L^{1}\left(\mathbb{H}^{n}\right) \longrightarrow L^{1, \infty}\left(\mathbb{H}^{n}\right)}=\left\|\mathbb{M}_{\gamma}^{c}\right\|_{L^{1}\left(\mathbb{H}^{n}\right) \longrightarrow L^{1, \infty}\left(\mathbb{H}^{n}\right)} .
$$

Theorem 14. For $1<p \leq \infty, \gamma>0$, we have

$$
\left\|\mathbb{M}^{c}\right\|_{L^{p}\left(\mathbb{H}^{n}\right) \longrightarrow L^{p}\left(\mathbb{H}^{n}\right)}=\left\|\mathbb{M}_{\gamma}^{c}\right\|_{L^{p}\left(\mathbb{H}^{n}\right) \longrightarrow L^{p}\left(\mathbb{H}^{n}\right)}
$$

Theorem 15. For $0<\lambda<n, \gamma>0$, we have

$$
\left\|\mathbb{M}^{c}\right\|_{\dot{M}^{l, \lambda}\left(\mathbb{H}^{n}\right) \longrightarrow \dot{W} M^{1, \lambda}\left(\mathbb{H}^{n}\right)}=\|\mathbb{M}\|_{\gamma}^{c} \|_{\dot{M}^{l, \lambda}\left(\mathbb{H}^{n}\right) \longrightarrow \dot{W} M^{1, \lambda}\left(\mathbb{H}^{n}\right)} .
$$

Theorem 16. For $1<p<\infty, 0<\lambda<n, \gamma>0$, we have

$$
\left\|\mathbb{M}^{c}\right\|_{\dot{M}^{p, \lambda}\left(\mathbb{H}^{n}\right) \longrightarrow \dot{M}^{p, \lambda}\left(\mathbb{H}^{n}\right)}=\|\mathbb{M}\|_{\gamma}^{c} \|_{\dot{M}^{p, \lambda}\left(\mathbb{H}^{n}\right) \longrightarrow \dot{M}^{p, \lambda}\left(\mathbb{H}^{n}\right)} .
$$

Remark 17. The four theorems only concern about equiva- lence of operator norms. We do not need that the norms are less than infinite, neither can we ensure that the norms are finite by our theorems. Readers can get results of upper bounds from such articles like [9], but the results are neither necessary nor sufficient with our theorems like Theorem 14 .

In the process of the proof, the general idea is that the norm of truncated maximal functions is less than that of the Hardy-Littlewood maximal function from definition. For some $\gamma$, the norm of the Hardy-Littlewood maximal function will be less than the norm of some truncated maximal functions from the definition of supremum. But according to Lemma 12, the norm of truncated maximal function is unrelated to $\gamma$. Thus, we can establish the equivalence of operator norms from that idea.

Proof of Theorem 18. By definition of truncated maximal functions, we always have $\mathbb{M}_{\gamma}^{c} f \leq \mathbb{M}^{c} f$ for any $\gamma>0$; combining with Proposition 1.1.3(1) of [3], we can obtain that

$$
\sup _{\lambda>0} \lambda\left(\lim _{\gamma \longrightarrow \infty} d_{\mathbb{M}_{\gamma}^{c} f}(\lambda)\right) \leq \sup _{\lambda>0} \lambda d_{\mathbb{M}^{c} f}(\lambda) .
$$

On the other hand, by Lemma 9, we have

$$
\sup _{\lambda>0} \lambda d_{\mathbb{M}^{c} f}(\lambda)=\sup _{\lambda>0} \lambda\left(\lim _{\gamma \longrightarrow \infty} d_{\mathbb{M}_{\gamma}^{c} f}(\lambda)\right) .
$$

For any $\varepsilon>0$, there exists $\lambda_{0}>0$, such that

$$
\sup _{\lambda>0} \lambda d_{\mathbb{M}^{c} f}(\lambda)-\varepsilon<\lambda_{0} d_{\mathbb{M}^{c} f}\left(\lambda_{0}\right)<\sup _{\lambda>0} \lambda d_{\mathbb{M}^{c} f}(\lambda) .
$$

\section{Obviously}

$$
\sup _{\lambda>0} \lambda\left(\lim _{\gamma \longrightarrow \infty} d_{\mathbb{M}_{\gamma}^{c} f}(\lambda)\right) \geq \lim _{\gamma \longrightarrow \infty} \lambda_{0} d_{\mathbb{M}_{\gamma}^{c} f}\left(\lambda_{0}\right) \geq \sup _{\lambda>0} \lambda d_{\mathbb{M}^{c} f}(\lambda)-\varepsilon .
$$

Then

$$
\sup _{\lambda>0} \lambda\left(\lim _{\gamma \longrightarrow \infty} d_{\mathbb{M}_{\gamma}^{c} f}(\lambda)\right) \geq \sup _{\lambda>0} \lambda d_{\mathbb{M}^{c} f}(\lambda) .
$$

So we obtain

$$
\lim _{\gamma \longrightarrow \infty} \sup _{\lambda>0} \lambda\left(d_{\mathbb{M}_{\gamma}^{c} f}(\lambda)\right)=\sup _{\lambda>0} \lambda d_{\mathbb{M}^{c} f}(\lambda) .
$$


Combining with Lemma 12, we have

$$
\begin{aligned}
\left\|\mathbb{M}^{c}\right\|_{L^{1}\left(\mathbb{H}^{n}\right) \longrightarrow L^{1, \infty}\left(\mathbb{H}^{n}\right)} & =\lim _{\gamma \longrightarrow \infty}\left\|\mathbb{M}_{\gamma}^{c}\right\|_{L^{1}\left(\mathbb{H}^{n}\right) \longrightarrow L^{1, \infty}\left(\mathbb{H}^{n}\right)} \\
& =\lim _{\gamma \longrightarrow \infty}\left\|\mathbb{M}_{1}^{c}\right\|_{L^{1}\left(\mathbb{H}^{n}\right) \longrightarrow L^{1, \infty}\left(\mathbb{H}^{n}\right)} \\
& =\left\|\mathbb{M}_{1}^{c}\right\|_{L^{1}\left(\mathbb{H}^{n}\right) \longrightarrow L^{1, \infty}\left(\mathbb{H}^{n}\right)} \\
& =\left\|\mathbb{M}_{\gamma}^{c}\right\|_{L^{1}\left(\mathbb{H}^{n}\right) \longrightarrow L^{1, \infty}\left(\mathbb{H}^{n}\right)} .
\end{aligned}
$$

So, we finish the proof.

Proof of Theorem 19. By definition of truncated maximal functions, we obtain that

$$
\left\|\mathbb{M}^{c}\right\|_{L^{p}\left(\mathbb{H}^{n}\right) \longrightarrow L^{p}\left(\mathbb{H}^{n}\right)} \geq\left\|\mathbb{M}_{\gamma}^{c}\right\|_{L^{p}\left(\mathbb{H}^{n}\right) \longrightarrow L^{p}\left(\mathbb{H}^{n}\right)} .
$$

Now, we only need to prove that

$$
\left\|\mathbb{M}^{c}\right\|_{L^{p}\left(\mathbb{H}^{n}\right) \longrightarrow L^{p}\left(\mathbb{H}^{n}\right)} \leq\left\|\mathbb{M}_{\gamma}^{c}\right\|_{L^{p}\left(\mathbb{H}^{n}\right) \longrightarrow L^{p}\left(\mathbb{H}^{n}\right)} .
$$

By Lemma 10, fixing $\varepsilon>0$, there exists $g \in C_{c}^{\infty}\left(\mathbb{H}^{n}\right)$, so that

$$
\frac{\left\|\mathbb{M}^{c} g\right\|_{L^{p}\left(\mathbb{H}^{n}\right)}}{\|g\|_{L^{p}\left(\mathbb{H}^{n}\right)}} \geq\left\|\mathbb{M}^{c}\right\|_{L^{p}\left(\mathbb{H}^{n}\right) \longrightarrow L^{p}\left(\mathbb{H}^{n}\right)}-\varepsilon
$$

Suppose that the support of $g$ is in the ball $\{x \in$ $\left.\mathbb{H}^{n}:|x|_{h}<R\right\}$.

Since $g$ has compact support, we can find $S>0$ such that

$$
\left\|\left(\mathbb{M}^{c} g\right)_{\chi\{|| \geq S\}}\right\|_{L^{p}\left(\mathbb{H}^{n}\right)} \leq \varepsilon\|g\|_{L^{p}\left(\mathbb{H}^{n}\right)} .
$$

Fix $\gamma_{0}>R+S$, when $|x|_{h}<S$,

$$
\mathbb{M}^{c} g(x)=\mathbb{M}_{\gamma_{0}}^{c} g(x) .
$$

So

$$
\begin{aligned}
\left\|\mathbb{M}_{\gamma_{0}}^{c} g\right\|_{L^{p}\left(\mathbb{H}^{n}\right)} & \geq\left\|\left(\mathbb{M}_{\gamma_{0}}^{c} g\right)_{\chi\{\mid \cdot \leq S\}}\right\|_{L^{p}\left(\mathbb{H}^{n}\right)}=\left\|\left(\mathbb{M}^{c} g\right)_{\chi\{\mid \cdot \leq S\}}\right\|_{L^{p}\left(\mathbb{H}^{n}\right)} \\
& \geq\left\|\mathbb{M}^{c} g\right\|_{L^{p}\left(\mathbb{H}^{n}\right)}-\left\|\left(\mathbb{M}^{c} g\right)_{\chi\{|\cdot| \geq S\}}\right\|_{L^{p}\left(\mathbb{H}^{n}\right)} \\
& \geq\left\|\mathbb{M}^{c}\right\|_{L^{p}\left(\mathbb{H}^{n}\right) \longrightarrow L^{p}\left(\mathbb{H}^{n}\right)}\|g\|_{L^{p}\left(\mathbb{H}^{n}\right)}-2 \varepsilon\|g\|_{L^{p}\left(\mathbb{H}^{n}\right)} .
\end{aligned}
$$

Then

$$
\left\|M_{1}^{c}\right\|_{L^{p}\left(\mathbb{H}^{n}\right) \longrightarrow L^{p}\left(\mathbb{H}^{n}\right)}=\left\|M_{\gamma_{0}}^{c}\right\|_{L^{p}\left(\mathbb{H}^{n}\right) \longrightarrow L^{p}\left(\mathbb{H}^{n}\right)} \geq\left\|M^{c}\right\|_{L^{p}\left(\mathbb{H}^{n}\right) \longrightarrow L^{p}\left(\mathbb{H}^{n}\right)}-2 \varepsilon
$$

For any $\gamma$, by Lemma 12

$$
\begin{aligned}
\left\|M_{\gamma}^{c}\right\|_{L^{p}\left(\mathbb{H}^{n}\right) \longrightarrow L^{p}\left(\mathbb{H}^{n}\right)} & =\left\|M_{1}^{c}\right\|_{L^{p}\left(\mathbb{H}^{n}\right) \longrightarrow L^{p}\left(\mathbb{H}^{n}\right)} \\
& \geq\left\|M^{c}\right\|_{L^{p}\left(\mathbb{H}^{n}\right) \longrightarrow L^{p}\left(\mathbb{H}^{n}\right)}-2 \varepsilon .
\end{aligned}
$$

So we can obtain (73).

Therefore, we have

$$
\left\|\mathbb{M}^{c}\right\|_{L^{p}\left(\mathbb{H}^{n}\right) \longrightarrow L^{p}\left(\mathbb{H}^{n}\right)}=\left\|\mathbb{M}_{\gamma}^{c}\right\|_{L^{p}\left(\mathbb{H}^{n}\right) \longrightarrow L^{p}\left(\mathbb{H}^{n}\right)} .
$$

Proof of Theorem 20. Using Lemmas 9 and 12, we directly compute that

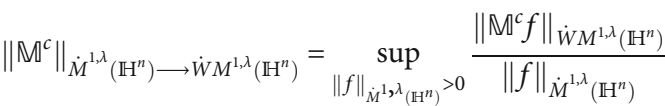

$$
\begin{aligned}
& =\sup _{\|f\|_{\hat{\mathcal{M}}^{1}, \lambda_{\left(\mathbb{H}^{n}\right)}>0}} \frac{\sup _{R>0}\left(1 / R^{\lambda}\right) \sup _{s>0} s\left|\left\{y \in B(0, R): \mathbb{M}^{c} f(y)>s\right\}\right|}{\|f\|_{\dot{M}^{1, \lambda}\left(\mathbb{H}^{n}\right)}}
\end{aligned}
$$

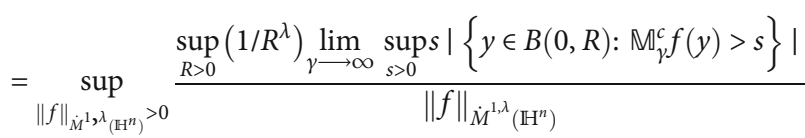

$$
\begin{aligned}
& =\lim _{\gamma \longrightarrow \infty} \sup _{\|f\|_{\hat{M}^{1}, \lambda_{\left(\mathbb{H}^{n}\right)}>0}} \frac{\sup _{R>0}\left(1 / R^{\lambda}\right) \sup _{s>0} s\left|\left\{y \in B(0, R): \mathbb{M}_{\gamma}^{c} f(y)>s\right\}\right|}{\|f\|_{\dot{M}^{1, \lambda}\left(\mathbb{H}^{n}\right)}} \\
& =\lim _{\gamma \longrightarrow \infty}\left\|\mathbb{M}_{\gamma}^{c}\right\|_{\dot{M}^{1, \lambda}\left(\mathbb{H}^{n}\right) \longrightarrow \dot{W} M^{1, \lambda}\left(\mathbb{H}^{n}\right)}=\left\|\mathbb{M}_{1}^{c}\right\|_{\dot{M}^{1, \lambda}\left(\mathbb{H}^{n}\right) \longrightarrow \dot{W} M^{1, \lambda}\left(\mathbb{H}^{n}\right)} \\
& =\left\|\mathbb{M}_{\gamma}^{c}\right\|_{\dot{M}^{1, \lambda}\left(\mathbb{H}^{n}\right) \longrightarrow \dot{W} M^{1, \lambda}\left(\mathbb{H}^{n}\right)} \text {. }
\end{aligned}
$$

We can immediately obtain Theorem 15 .

Proof of Theorem 21. By definition of truncated maximal functions, we obtain that

$$
\left\|\mathbb{M}^{c}\right\|_{\dot{M}^{p, \lambda}\left(\mathbb{H}^{n}\right) \longrightarrow \dot{M}^{p, \lambda}\left(\mathbb{H}^{n}\right)} \geq\|\mathbb{M}\|_{\dot{M}^{p, \lambda}\left(\mathbb{H}^{n}\right) \longrightarrow \dot{M}^{p, \lambda}\left(\mathbb{H}^{n}\right)} .
$$

It is remained to be proved that

$$
\left\|\mathbb{M}^{c}\right\|_{\dot{M}^{p, \lambda}\left(\mathbb{H}^{n}\right) \longrightarrow \dot{M}^{p, \lambda}\left(\mathbb{H}^{n}\right)} \leq\|\mathbb{M}\|_{\gamma}^{c} \|_{\dot{M}^{p, \lambda}\left(\mathbb{H}^{n}\right) \longrightarrow \dot{M}^{p, \lambda}\left(\mathbb{H}^{n}\right)}
$$


Using distribution functions and Lemma 9, we have

$$
\begin{aligned}
& \frac{1}{R^{\lambda}} \int_{B(0, R)}\left|\mathbb{M}^{c} f(x)\right|^{p} d x=\frac{1}{R^{\lambda}}\left\|\mathbb{M}^{c} f \chi_{B(0, R)}\right\|_{L^{p}\left(\mathbb{H}^{n}\right)}^{p} \\
& \quad=\frac{p}{R^{\lambda}} \int_{0}^{\infty} \alpha^{p-1}\left|\left\{x \in B(0, R):\left|\mathbb{M}^{c} f(x)\right|>\alpha\right\}\right| d \alpha \\
& \quad=\frac{p}{R^{\lambda}} \int_{0}^{\infty} \alpha^{p-1} \lim _{\gamma \longrightarrow \infty}\left|B(0, R) \cap\left\{x \in \mathbb{H}^{n}:\left|\mathbb{M}_{\gamma}^{c} f(x)\right|>\alpha\right\}\right| d \alpha \\
& \quad=\lim _{\gamma \longrightarrow \infty} \frac{p}{R^{\lambda}} \int_{0}^{\infty} \alpha^{p-1}\left|B(0, R) \cap\left\{x \in \mathbb{H}^{n}:\left|\mathbb{M}_{\gamma}^{c} f(x)\right|>\alpha\right\}\right| d \alpha \\
& \quad=\lim _{\gamma \longrightarrow \infty} \frac{1}{R^{\lambda}} \int_{B(0, R)}\left|\mathbb{M}_{\gamma}^{c} f(x)\right|^{p} d x .
\end{aligned}
$$

Taking the supremum over $R$, combining with Lemma 12 , we have

$$
\begin{aligned}
\left\|\mathbb{M}^{c}\right\|_{\dot{M}^{p, \lambda}\left(\mathbb{H}^{n}\right)} & \leq \lim _{\gamma \longrightarrow \infty}\left\|\mathbb{M}_{\gamma}^{c}\right\|_{\dot{M}^{p, \lambda}\left(\mathbb{H}^{n}\right)} \\
& =\lim _{\gamma \longrightarrow \infty}\left\|\mathbb{M}_{\gamma}^{c}\right\|_{\dot{M}^{p, \lambda}\left(\mathbb{H}^{n}\right) \longrightarrow \dot{M}^{p, \lambda}\left(\mathbb{H}^{n}\right)}\|f\|_{\dot{M}^{p, \lambda}\left(\mathbb{H}^{n}\right)} \\
& =\left\|\mathbb{M}_{1}^{c}\right\|_{\dot{M}^{p, \lambda}}\left(\mathbb{H}^{n}\right) \longrightarrow \dot{M}^{p, \lambda}\left(\mathbb{H}^{n}\right) \\
& =\|f\|_{\dot{M}^{p, \lambda}\left(\mathbb{H}^{n}\right)} \\
& \mathbb{M}_{\gamma}^{c}\left\|_{\dot{M}^{p, \lambda}\left(\mathbb{H}^{n}\right) \longrightarrow \dot{M}^{p, \lambda}\left(\mathbb{H}^{n}\right)}\right\| f \|_{\dot{M}^{p, \lambda}\left(\mathbb{H}^{n}\right)} .
\end{aligned}
$$

So we obtain (83).

Then

$$
\left\|\mathbb{M}^{c}\right\|_{\dot{M}^{p, \lambda}\left(\mathbb{H}^{n}\right) \longrightarrow \dot{M}^{p, \lambda}\left(\mathbb{H}^{n}\right)}=\left\|\mathbb{M}_{\gamma}^{c}\right\|_{\dot{M}^{p, \lambda}\left(\mathbb{H}^{n}\right) \longrightarrow \dot{M}^{p, \lambda}\left(\mathbb{H}^{n}\right)}
$$

So the proof is accomplished.

We only present the details of centered maximal function and its truncation. In fact, for uncentered case, there still holds similar results:

\section{Theorem 22.}

(1) For $\gamma>0$, we have

$$
\|\mathbb{M}\|_{L^{1}\left(\mathbb{H}^{n}\right) \longrightarrow L^{1, \infty}\left(\mathbb{H}^{n}\right)}=\left\|\mathbb{M}_{\gamma}\right\|_{L^{1}\left(\mathbb{H}^{n}\right) \longrightarrow L^{1, \infty}\left(\mathbb{H}^{n}\right)} .
$$

(2) For $1<p \leq \infty, \gamma>0$, we have

$$
\|\mathbb{M}\|_{L^{p}\left(\mathbb{H}^{n}\right) \longrightarrow L^{p}\left(\mathbb{H}^{n}\right)}=\left\|\mathbb{M}_{\gamma}\right\|_{L^{p}\left(\mathbb{H}^{n}\right) \longrightarrow L^{p}\left(\mathbb{H}^{n}\right)} .
$$$$
\left\|\mathbb{M}_{\gamma_{1}, \gamma_{2}}\right\|_{\dot{M}^{p, \lambda}\left(\mathbb{H}^{n}\right) \longrightarrow \dot{M}^{p, \lambda}\left(\mathbb{H}^{n}\right)}=\left\|\mathbb{M}_{\gamma_{3}, \gamma_{4}}\right\|_{\dot{M}^{p, \lambda}\left(\mathbb{H}^{n}\right) \longrightarrow \dot{M}^{p, \lambda}\left(\mathbb{H}^{n}\right)} .
$$

$$
\|\mathbb{M}\|_{\dot{M}^{1, \lambda}\left(\mathbb{H}^{n}\right) \longrightarrow \dot{W} M^{1, \lambda}\left(\mathbb{H}^{n}\right)}=\left\|\mathbb{M}_{\gamma}\right\|_{\dot{M}^{1, \lambda}\left(\mathbb{H}^{n}\right) \longrightarrow \dot{W} M^{1, \lambda}\left(\mathbb{H}^{n}\right)}
$$

(4) For $1<p<\infty, 0<\lambda<n, \gamma>0$, we have

$$
\|\mathbb{M}\|_{\dot{M}^{p, \lambda}}\left(\mathbb{H}^{n}\right) \longrightarrow \dot{M}^{p, \lambda}\left(\mathbb{H}^{n}\right)=\left\|\mathbb{M}_{\gamma}\right\|_{\dot{M}^{p, \lambda}\left(\mathbb{H}^{n}\right) \longrightarrow \dot{M}^{p, \lambda}\left(\mathbb{H}^{n}\right)}
$$

We save the details since the methods are the same.

Remark 23. For general cases of truncated maximal function, we also can define them on the Heisenberg group:

$$
\mathbb{M}_{\alpha, \beta} f(x)=\sup _{\alpha<r<\beta} \frac{1}{m(B(x, r))} \int_{B(x, r)}|f(y)| d m(y) .
$$

Using the same methods, we can prove such theorem:

Theorem 24. $1<p \leq \infty . \gamma_{1}, \gamma_{2}, \gamma_{3}, \gamma_{4}$ is positive number, and

$$
\frac{\gamma_{1}}{\gamma_{2}}=\frac{\gamma_{3}}{\gamma_{4}}=C
$$

Then, the norms of $\mathbb{M}_{\gamma_{1}, \gamma_{2}}$ and $\mathbb{M}_{\gamma_{3}, \gamma_{4}}$ have such relations: (1)

$$
\left\|\mathbb{M}_{\gamma_{1}, \gamma_{2}}\right\|_{L^{1}\left(\mathbb{H}^{n}\right) \longrightarrow L^{1, \infty}\left(\mathbb{H}^{n}\right)}=\left\|\mathbb{M}_{\gamma_{3}, \gamma_{4}}\right\|_{L^{1}\left(\mathbb{H}^{n}\right) \longrightarrow L^{1, \infty}\left(\mathbb{H}^{n}\right)} .
$$

(2) For $1<p \leq \infty$

$$
\left\|\mathbb{M}_{\gamma_{1}, \gamma_{2}}\right\|_{L^{p}\left(\mathbb{H}^{n}\right) \longrightarrow L^{p}\left(\mathbb{H}^{n}\right)}=\left\|\mathbb{M}_{\gamma_{3}, \gamma_{4}}\right\|_{L^{p}\left(\mathbb{H}^{n}\right) \longrightarrow L^{p}\left(\mathbb{H}^{n}\right)} .
$$

(3) For $0<\lambda<n$, we have

$$
\left\|\mathbb{M}_{\gamma_{1}, \gamma_{2}}\right\|_{\dot{M}^{1, \lambda}\left(\mathbb{H}^{n}\right) \longrightarrow \dot{W} M^{1, \lambda}\left(\mathbb{H}^{n}\right)}=\left\|\mathbb{M}_{\gamma_{3}, \gamma_{4}}\right\|_{\dot{M}^{1, \lambda}\left(\mathbb{H}^{n}\right) \longrightarrow \dot{W} M^{1, \lambda}\left(\mathbb{H}^{n}\right)} .
$$

(4) For $1<p<\infty, 0<\lambda<n$, we have

The main theorems can be seen as the special case $C=0$. 


\section{Data Availability}

This is a mathematical paper, our work is mainly reasoning, and we only used some definitions and theorems in the manuscript as the basis of our research, which can be found in the references we give; most of these concepts are common in mathematics. On the other hand, our manuscript is closely related to Xingsong Zhang's another work (Zhang, Xingsong; Wei, Mingquan; Yan, Dunyan; and He, Qianjun; Equivalence of Operator Norm for Hardy-Littlewood Maximal Operators and Their Truncated Operators on Morrey Spaces. Front. Math. China 15 (2020), no. 1, 215-223. 42B25 (42B20); https://ink.springer.com/article/10.1007/s11464-020-08126). We consider similar problem in different space.

\section{Conflicts of Interest}

The authors declare that they have no conflicts of interest.

\section{References}

[1] N. G. Albuquerque and L. Rezende, "Asymptotic estimates for unimodular multilinear forms with small norms on sequence spaces," Bulletin of the Brazilian Mathematical Society, vol. 52, no. 1, pp. 23-39, 2021.

[2] B. Di, Q. He, and D. Yan, "Some weighted estimates on gaussian measure spaces," Bulletin of the Malaysian Mathematical Sciences Society, 2021, In press.

[3] L. Grafakos, Classical and Modern Fourier Analysis, China Machine Press, Beijing, 2005.

[4] V. S. Guliyev, M. N. Omarova, M. A. Ragusa, and A. Scapellato, "Regularity of solutions of elliptic equations in divergence form in modified local generalized Morrey spaces," Analysis and Mathematical Physics, vol. 11, no. 1, 2021.

[5] E. M. Stein, Harmonic Analysis, Real Variable Methods, Orthogonality, and Osciallatory Integrals, Princeton University Press, New Jersey, 1993.

[6] A. Melas, "The best constant for the centered HardyLittlewood maximal inequality," Annals of Mathematics, vol. 157, no. 2, pp. 647-688, 2003.

[7] M. Wei, X. Nie, D. Wu, and D. Yan, "A note on HardyLittlewood maximal operators," Journal of the Academy of Nutrition and Dietetics, vol. 2016, no. 1, 2016.

[8] X. Zhang, M. Wei, D. Yan, and Q. He, "Equivalence of operator norm for Hardy-Littlewood maximal operators and their truncated operators on Morrey spaces," Frontiers of Mathematics in China, vol. 15, no. 1, pp. 215-223, 2020.

[9] H. Li and B. Qian, "Centered Hardy-Littlewood maximal functions on Heisenberg type groups," Transactions of the American Mathematical Society, vol. 366, no. 3, pp. 1497-1524, 2014.

[10] W. Rudin, Real and Complex Analysis, McGraw-Hill Publishing Co., New York, 1987. 